\title{
A new Very Large Eddy Simulation model for simulation of turbulent flow
}

\author{
Xingsi Han and Siniša Krajnović
}

\begin{abstract}
Among various hybrid RANS/LES methodologies, Speziale's Very Large Eddy Simulation (VLES) is one that was early proposed and is a unified simulation approach that can change seamlessly from RANS to DNS depending on the numerical resolution. The present study proposes a new improved variant of the original VLES model. The advantages are achieved in two ways: (1) RANS simulation can be recovered near the wall which is similar to the Detached Eddy Simulation (DES) concept; (2) An LES subgrid scale model can be reached by the introduction of a third length scale, i.e. integral turbulence length scale. Thus the new model can provide a proper LES mode between the RANS and DNS limits. This new methodology is implemented in the standard $k-\varepsilon$ model and Wilcox's $k-\omega$ model. Applications are conducted for the turbulent channel flow at $R e_{\tau}=395$ and turbulent flow past a square cylinder at $R e=22000$. Results are compared with previous studies. It is demonstrated that the new method is quite effective in resolving the large flow structures, and can give satisfactory predictions on a very coarse mesh.
\end{abstract}

\section{Introduction}

In many industrial and engineering applications, the Reynolds-Averaged NavierStokes (RANS) approach is still the dominant method for simulating turbulent flows at high Reynolds number. However, the RANS method performs poorly in complex unsteady flows that are dominated by coherent large-eddy structures. Large Eddy Simulation (LES) can resolve the large structures accurately, as the unsteady largescale turbulent motions are explicitly resolved in the LES method. Unfortunately, LES is often not computationally feasible, as it suffers from a very restrictive grid resolution requirement near the wall. An idea, namely hybrid RANS/LES methodol-

Xingsi Han and Siniša Krajnović

Division of Fluid Dynamics, Department of Applied Mechanics, Chalmers University of Technology, 41296 Gothenburg, Sweden, e-mail: xingsiechalmers.se, sinisa@chalmers. se 
ogy, pursued by many researchers is to switch or gradually blend to a RANS method near the wall. The underlying concept is to combine the computational efficiency of RANS for modeling the flow in the near-wall regions, with the accurate LES method for simulating the large-scale turbulent structures in the regions away from the wall.

Speziale was among the first to propose a hybrid method that combines the advantages of different turbulence approaches [18]. This approach was later called Flow Simulation Methodology (FSM) [4, 6] and has shown robustness in some applications. In this approach, a generalized turbulence model is obtained by rescaling a conventional RANS model through the introduction of a resolution control function $F_{r}$, i.e. the subscale turbulent stress tensor is modeled by damping the Reynolds stresses, that is

$$
\tau_{i j}^{s u b}=F_{r} \tau_{i j}^{R A N S}
$$

in which the resolution control function $F_{r}$ has the form

$$
F_{r}=\left[1.0-\exp \left(\frac{-\beta \Delta}{L_{k}}\right)\right]^{n}
$$

where $\beta \sim O\left(10^{-3}\right), n \sim O(1)$ are some modelling (unspecified) parameters, $\Delta$ is the representative mesh spacing (cutoff length scale) and, $L_{k}$ is the Kolmogorov length scale defined as $L_{k}=v^{\frac{3}{4}} / \varepsilon^{\frac{1}{4}}$. In the limit such as $\Delta / L_{k} \rightarrow 0$, all relevant scales are resolved (e.g. $\tau_{i j}^{s u b}=0$ ), i.e. the model approaches to a DNS method. The regular RANS behavior is recovered (e.g. $\tau_{i j}^{s u b}=\tau_{i j}^{R A N S}$ ) at the other limit as $\Delta / L_{k} \rightarrow \infty$ as the mesh becomes coarse. It is considered a VLES methodology between the two limits.

However, the model damps the Reynolds stress too much, and it is nearly impossible to recover to a RANS simulation unless the mesh is unreasonably coarse [20]. The model therefore needs quite fine mesh resolutions near the wall as does a LES method and does not work effectively for wall-bounded flows. Furthermore, there are a number of issues that were never completely specified by Speziale (please see [16]). One important issue is that properly reaching both the DNS and RANS limits in this model does not guarantee that the corresponding approach provides a correct LES mode. As pointed out by Sagaut et al. [16], when the Reynolds number tends to infinity (i.e. $L_{k} \rightarrow 0$ ), this model systematically gives a RANS behavior according to Eq. 2, which means that the grid spacing no longer has any influence on the eddy viscosity and an LES subgrid scale cannot be reached regardless of how fine the grid is.

Several other approaches follow Speziale's method, such as the Limited Numerical Scales (LNS) approach by Batten et al. [1], the Partially Resolved Numerical Simulation (PRNS) by Liu and Shih [9], and a newly developed approach by Hsieh et al. [5]. The present paper uses the VLES acronym to refer generically to all these, similar, strategies. The objective of the present study is to try to make an improvement with respect to the two disadvantages of the original Speziale's method mentioned above. The new model's performance is validated in the application for 
two classical flows, fully-developed turbulent channel flow and turbulent flow past a square cylinder at $R e=22000$.

\section{Mathematical formulation and numerical detail}

In the VLES concept, the subscale stress is rescaled by a resolution control function, $F_{r}$, whose value lies between zero and one. The predictive accuracy of VLES depends on $F_{r}$ and the specific RANS turbulence model. The present study focuses on the more important issue of formulating the $F_{r}$ control function. According to Hsieh et al. [5], a generalized functional form of $F_{r}$ can be written based on the turbulence energy spectrum in the form of

$$
F_{r}=\frac{\int_{L_{k}}^{L_{c}} E(L) d L}{\int_{L_{k}}^{L_{i}} E(L) d L}
$$

in which $L_{c}, L_{i}$ and $L_{k}$ are the turbulent cutoff length scale, integral length scale and Kolmogorov length scale, respectively, defined as

$$
L_{c}=C_{x}\left(\Delta_{x} \Delta_{y} \Delta_{z}\right)^{\frac{1}{3}} \quad L_{i}=k^{\frac{3}{2}} / \varepsilon \quad L_{k}=v^{\frac{3}{4}} / \varepsilon^{\frac{1}{4}}
$$

where the definite integrals in Eq. 3 represent the turbulent kinetic energy between $L_{k}$ and $L_{c}$, and between $L_{k}$ and $L_{i}$, and therefore roughly resemble the ratio of the unresolved turbulent kinetic energy to the total turbulent kinetic energy. Following this idea, a new formulation of $F_{r}$ can be obtained based on the original Speziale's model. Assuming Eq. 2 is suitable for the inertial sub-range scales, it can be gotten that

$$
\begin{gathered}
F_{r}=\frac{\tau_{i i}\left(L_{k} \rightarrow L_{c}\right)}{\tau_{i i}\left(L_{k} \rightarrow L_{i}\right)}=\frac{\left[1.0-\exp \left(-\beta L_{c} / L_{k}\right)\right]^{n} \tau_{i j}^{R A N S}}{\left[1.0-\exp \left(-\beta L_{i} / L_{k}\right)\right]^{n} \tau_{i j}^{R A N S}} \\
F_{r}=\left[\left(1.0-\exp \left(-\beta L_{c} / L_{k}\right)\right) /\left(1.0-\exp \left(-\beta L_{i} / L_{k}\right)\right)\right]^{n}
\end{gathered}
$$

Eq. 5 is the proposed generalized functional form of $F_{r}$. Unfortunately, for $L_{c}>$ $L_{i}$, Eq. 5 leads to $F_{r}>1.0$, which is unphysical. To ensure $F_{r}$ having a value between 0 and 1.0, we propose the final form of the new model to be

$$
F_{r}=\min \left(1.0,\left[\left(1.0-\exp \left(-\beta L_{c} / L_{k}\right)\right) /\left(1.0-\exp \left(-\beta L_{i} / L_{k}\right)\right)\right]^{n}\right)
$$

where the $\min (x, y)$ refers to the minimum value between $x$ and $y$. There are three model parameters in the new model, $C_{x}$ in Eq. 4 , and $\beta$ and $n$ that come from the 
original Speziale's model. To calibrate the model constant $C_{x}$, we follow the idea of Johansen et al. [7] who assume that the standard $k-\varepsilon$ model becomes identical to the Smagorinsky LES model when $L_{c}=L_{i}$. In this situation, the model constant $C_{x}$ is related to the Smagorinsky LES model constant $C_{s}$ as

$$
C_{x}=\sqrt{0.3} C_{s} / C_{\mu}
$$

where $C_{\mu}=0.09$ is the model constant in the standard $k-\varepsilon$ model. As the typical Smagorinsky LES model constant $C_{S}$ has a value of 0.1 , we can finally get the model constant $C_{x}=0.61$.

It can be seen from Eq. 5 that in the limit of very fine mesh resolution, i.e. when the modeled kinetic energy approaches zero, Eq. 5 can be expressed in another form using the Taylor series

$$
F_{r} \rightarrow\left[\left(-\beta L_{c} / L_{k}\right) /\left(-\beta L_{i} / L_{k}\right)\right]^{n}=\left(\frac{L_{c}}{L_{i}}\right)^{n}
$$

which has exactly the same form as in several previous hybrid RANS/LES methodologies (please see [16]). The functional form of Eq. 8 actually implies that the hybrid methodology approaches a LES method with very fine mesh resolution. In addition, in those methods, model parameter $n$ has a fixed value, although two different values exist, i.e. $n=4 / 3$ ( $[12,8])$ and $n=2$ ( [15]). On the basis of this, we propose to use a model constant of $n=4 / 3$ or $n=2$.

Equations 1 and 6 constitute the new proposed VLES model. The model constants are $C_{x}=0.61, n=4 / 3$ or $n=2$, and the recommended value of $\beta$ is $\beta=2.0 \times 10^{-3}$ based on the studies of Speziale [18] and Fasel et al. [4]. It should be noted that near the wall, $L_{c}>L_{i}$ leading to $F_{r}=1$ (see Eq. 6); the hybrid model recovers to the RANS model, similar to the DES concept.

As in the original VLES model, the new model can be blended with any trusted RANS turbulence model. For the initial study here, it was implemented in the standard $k-\varepsilon$ model and Wilcox's $k-\omega$ model. There are actually several different methods that can be implemented in the hybrid method based on the RANS method. The present study adopts a simple one following the ideas of the PRNS method [9], the LNS method [1] and from [5], in which only the formulation of the turbulent viscosity is modified, in the form of

$$
\mu_{t}^{s u b}=F_{r} \mu_{t}^{R A N S}
$$

and the governing equations of turbulence quantities keep exactly the same forms as in the original RANS turbulence model. The implementations in the standard $k-\varepsilon$ model and Wilcox's $k-\omega$ model are given in the appendix. It should be noted that, in the framework of the $k-\omega$ model, the computation of length scales in Eq. 4 is accomplished by using the relation between $\omega$ and $\varepsilon$, i.e. $\varepsilon=0.09 k \omega$.

The new VLES models were implemented in the FLUENT commercial CFD software. The convective terms are discretized using a second-order central differencing scheme for channel flow and a bounded central differencing scheme for 
flow past a square cylinder. The second-order upwind scheme was used for the turbulence model equations. The temporal advancement was approximated using a second-order implicit scheme. The SIMPLEC algorithm was used for pressurevelocity coupling.

\section{Results of turbulent channel flow}

The first test case is a fully developed turbulent channel flow at $R e_{\tau}=\delta u_{\tau} / \mathrm{v}=$ 395 which was studied using DNS [13]. It was selected to highlight the feasibility of the VLES model to simulate near-wall turbulence. The computational domain is $2 \pi \delta \times 2 \delta \times \pi \delta$. Two different, relatively coarse, meshes were used, i.e. mesh1 $(32 \times 64 \times 32)$ and mesh $2(40 \times 80 \times 40)$. The mesh is clustered near the wall and the first node is located at $y^{+} \approx 1.0$.

Table 1 Comparisons of friction velocity, $u_{\tau}$, between different models

\begin{tabular}{ccccccc}
\hline mesh1 & DNS [13] & $V L E S-k \varepsilon^{n 1}$ & $V L E S-k \varepsilon^{n 2}$ & $V L E S-k \omega^{n 2}$ & VLES-org. & LES-WALE \\
\hline$u_{\tau}$ & 1.0 & 1.015 & 0.975 & 0.899 & 0.824 & 0.820 \\
\hline \hline mesh2 & DNS [13] & $V L E S-k \varepsilon^{n 2}$ & $V L E S-k \omega^{n 2}$ & VLES-org. & LES-WALE & \\
\hline$u_{\tau}$ & 1.0 & 0.985 & 0.924 & 0.876 & 0.869 \\
\hline
\end{tabular}

The computed friction velocities are compared in Table 1 , where superscript $n 1$ refers to the use of the model constant $n=4 / 3$ and $n 2$ refers to the use of $n=2$ in Eq. 6, VLES - org. refers to the original Speziale's model, and LES - WALE refers to the simulation using the LES WALE model [14]. It can be seen that the new VLES models clearly improve the results, compared with the original VLES model and LES model. The VLES- $k \varepsilon$ models predict quite good results compared with DNS.

The mean streamwise velocity and RMS velocity profiles by different models are compared in Fig. 1. The first observation is that the new VLES model using the model constant of $n=2$ predicts obviously better results than using $n=4 / 3$. Therefore, in the following sections, the results shown are all obtained using the model constant of $n=2$. The new VLES models based on $k-\varepsilon$ and $k-\omega$ models both improve the results compared with the original VLES model and with the LES WALE model. It should be noted that predictions made by the original VLES model and LES WALE are quite close in all velocity profiles on the same mesh. The new VLES model based on the $k-\varepsilon$ model gives slightly better results than the one based on the $k-\omega$ model, which can be observed in Table 1. It can also be seen that, with increasing mesh resolution, the predictions of the new VLES models are improved. However, the level of improvement is dependent on the underlaying RANS 

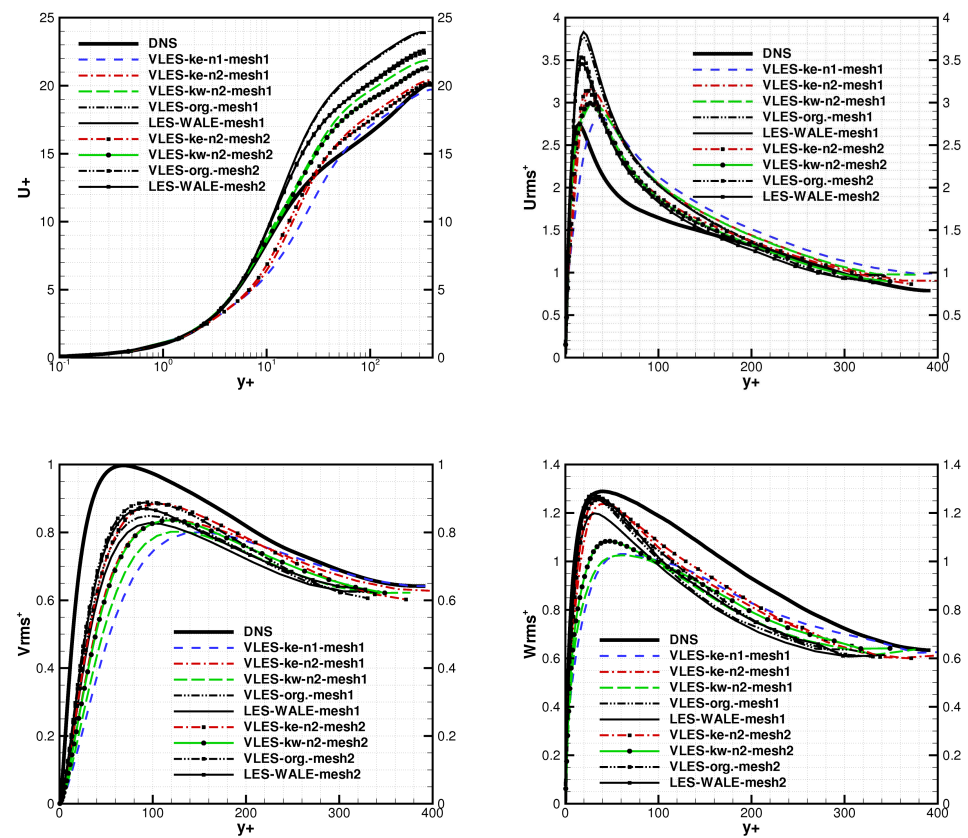

Fig. 1 Comparisons of velocity and RMS velocity profiles for channel flow

turbulence model. Compared with VLES based on the $k-\varepsilon$ model, the VLES based on the $k-\omega$ is more sensitive to the mesh resolution. It seems that the new VLES based on the $k-\varepsilon$ model is quite insensitive to the mesh resolution, which means that it can resolve quite reasonable results on a very coarse mesh. This feature can also be observed in the next case of flow past a square cylinder.

\section{Results of turbulent flow past a square cylinder}

The new VLES model is also applied for the flow past a square cylinder at $R e=$ 22000 based on the cylinder edge length $D$. The square cylinder is aligned in the $z$ (spanwise) direction and the inlet flow is set in the $x$ (streamwise) direction. The computational domain is $20 D \times 14 D \times 4 D$. The lateral dimension $14 D$ is the same as in Lyn's experiment [11], and the lateral boundaries are also subject to the wall boundary conditions to make the comparison with experiment more appropriate. Two different coarse meshes are used. The grid is clustered near the wall and the first node is located around $y^{+}=1.0$. The first mesh is quite coarse with a resolution of $85 \times 60 \times 10$ (about 0.048 million cells in total), and the second by refining the first mesh near the square cylinder (within $2.0 D$ ), results in a mesh containing about 
0.144 million cells. This is still very coarse, compared with the LES studies in [17], which uses a mesh of about 0.485 million and 1.066 millon cells, and the DES studies in [2], which uses a mesh with 8.467 million cells. The flow was simulated by both the new VLES models based on the $k-\varepsilon$ model and $k-\omega$ model respectively using the model constant of $n=2$. A non-dimensional time step, $t^{*}=t U_{\text {inlet }} / D$ equal to 0.01 , was used for all simulations.

Table 2 Comparisons of global flow parameters between different models

\begin{tabular}{cccccc}
\hline case & $R e / 10^{3}$ & $S t$ & $C_{D \_ \text {mean }}$ & $C_{D \_ \text {rms }}$ & $C_{\text {L_mean }}$ \\
\hline VLES $-k \varepsilon^{n 2}-$ mesh 1 & 22 & 0.139 & 2.27 & 0.352 & 1.126 \\
$V L E S-k \omega^{n 2}-$ mesh 1 & 22 & 0.130 & 2.30 & 0.362 & 1.176 \\
$V L E S-k \varepsilon^{n 2}-$ mesh 2 & 22 & 0.126 & 2.30 & 0.230 & 1.400 \\
$V L E S-k \omega^{n 2}-$ mesh 2 & 22 & 0.124 & 2.33 & 0.277 & 1.394 \\
LES [17] & 22 & $0.126-0.132$ & $2.03-2.32$ & $0.16-0.20$ & $1.23-1.54$ \\
DES (fine) [2] & 19.4 & 0.125 & 2.11 & 0.26 & 1.16 \\
Exp. Lyn [11] & 21.4 & 0.132 & 2.1 & - & - \\
Exp. Durao [3] & 14 & 0.138 & - & - & - \\
Exp. Luo [10] & 34 & 0.13 & 2.2 & 0.18 & 1.2 \\
\hline
\end{tabular}

The global parameters of the flow fields are compared in Table 2 with some previous studies. Although both meshes used are very coarse compared with previous numerical studies, all the global parameters predicted are acceptable. On the finer mesh, the results of RMS drag and lift coefficients are obviously improved. However, the Strouhal number is slightly underpredicted and the mean drag coefficient overpredicted; this might resulted from the mesh used in the present study being very coarse. Previous DES studies [2] also found that the Strouhal number was underpredicted and the mean drag coefficient overpredicted when the mesh was not fine enough. The results also demonstrate that the new VLES model based on the $k-\varepsilon$ model is slightly better than the one based on the $k-\omega$ model.

The averaged and RMS velocities along the central line are compared in Fig. 2, and the averaged velocities at location $x / D=1.0$ are shown in Fig. 3. The new VLES predicts quite reasonable results on the quite coarse meshes compared with the LES results obtained in a dynamics Smagorinsky model in [17], and experimental data of Lyn et al. [11]. On the finer mesh, the VLES predicts better velocity distributions, especially for the RMS velocities. The results of the two VLES models are quite close based on the $k-\varepsilon$ model and $k-\omega$ model, except for the streamwise RMS velocity predictions. It can be seen that the $U$ velocities are quite higher than the experiments, but better than the LES results [17]. Previous simulations found that the velocity is quite hard to be accurately predicted unless the mesh is very fine. Considering that the finer mesh used for VLES simulations is much coarser than the DES study [2] (the total cell number used is about 1.7\% of that in DES study), this might result in the overprediction of $U$ velocity. The underprediction of $W_{r m s}$ may also resulted from the coarse mesh as only 10 girds were placed in the spanwise direction. The performances of the VLES model on a finer mesh (around 1.0 million 

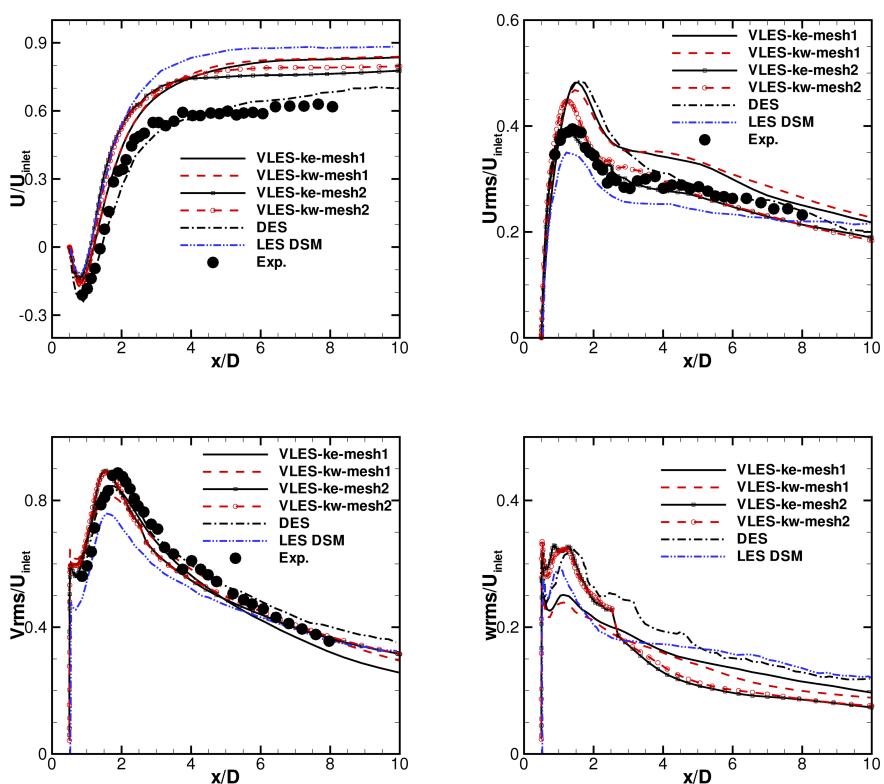

Fig. 2 Comparisons of velocity and RMS velocity profiles along the central line including the LES results [17], DES results [2] and experiments [11]

cells) should be conducted later. However, these results still demonstrate that the new VLES model is quite efficient to resolve the flow field, and that comparative results can be obtained using much coarser computational meshes than in previous LES computations.
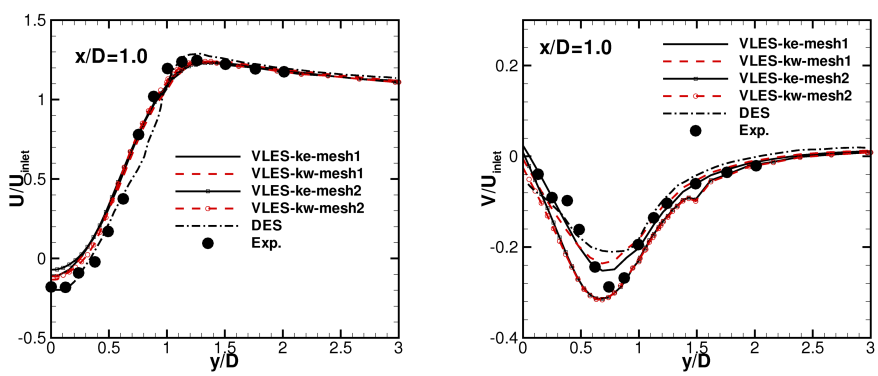

Fig. 3 Comparisons of streamwise and transverse velocity profiles at $x / D=1.0$ including $D E S$ results [2] and experiments [11] 


\section{Conclusions}

A new Very Large Eddy Simulation (VLES) method was proposed in the present work that is based on Speziale's VLES method. The new improved method can recover to a RANS simulation near the wall and also provide a proper LES mode between the limits of RANS and DNS. It was implemented in the standard $k-$ $\varepsilon$ model and Wilcox's $k-\omega$ model, and applied for turbulent channel flow and flow past a square cylinder. The results are compared with those of several previous studies. It is found that the new method is quite effective in resolving the large flow structures in both flow cases, and can give satisfactory predictions on a very coarse mesh compared with LES study, in both implementations based on the $k-\varepsilon$ model and the $k-\omega$ model. It also seems that the new model is not sensitive to the mesh resolution, which implies that acceptable results can be obtained on very coarse meshes using the new model, which is an obvious advantage in complex engineering applications.

\section{Appendix 1: VLES based on the standard $k-\varepsilon$ model}

The modeled transport equations for $k$ and $\varepsilon$ are exactly the same as in the standard $k-\varepsilon$ model given by

$$
\begin{gathered}
\frac{D \rho k}{D t}=P_{k}-\rho \varepsilon+\frac{\partial}{\partial x_{j}}\left[\left(\mu+\frac{\mu_{t}}{\sigma_{k}}\right) \frac{\partial k}{\partial x_{j}}\right] \\
\frac{D \rho \varepsilon}{D t}=\frac{\varepsilon}{k}\left(C_{\varepsilon 1} P_{k}-C_{\varepsilon 2} \rho \varepsilon\right)+\frac{\partial}{\partial x_{j}}\left[\left(\mu+\frac{\mu_{t}}{\sigma_{\varepsilon}}\right) \frac{\partial \varepsilon}{\partial x_{j}}\right] \\
\mu_{t}=F_{r} \rho C_{\mu} k^{2} / \varepsilon
\end{gathered}
$$

The model constants are also exactly the same as in the standard $k-\varepsilon$ model. Function $F_{r}$ is shown in Eq. 6 and the length scales are given in Eq. 4.

\section{Appendix 2: VLES based on Wilcox's $k-\omega$ model}

The modeled transport equations for $k$ and $\omega$ are exactly the same as in the Wilcox's $k-\omega$ model, given by

$$
\begin{gathered}
\frac{D \rho k}{D t}=P_{k}-\rho \beta_{0}^{*} f_{\beta^{*} k \omega}+\frac{\partial}{\partial x_{j}}\left[\left(\mu+\frac{\mu_{t}}{\sigma_{k}}\right) \frac{\partial k}{\partial x_{j}}\right] \\
\frac{D \rho \omega}{D t}=\alpha \frac{\omega}{k} P_{k}-\rho \beta_{0} f_{\beta} \omega^{2}+\frac{\partial}{\partial x_{j}}\left[\left(\mu+\frac{\mu_{t}}{\sigma_{\omega}}\right) \frac{\partial \omega}{\partial x_{j}}\right]
\end{gathered}
$$




$$
\mu_{t}=F_{r} \rho k / \omega
$$

The model constants are also exactly the same as in the Wilcox's $k-\omega$ model (see [19] for details). Function $F_{r}$ is shown in Eq. 6. The length scales are calculated in the framework of the $k-\omega$ model as

$$
L_{c}=C_{x}\left(\Delta_{x} \Delta_{y} \Delta_{z}\right)^{\frac{1}{3}} \quad L_{i}=k^{\frac{3}{2}} / \varepsilon \quad L_{k}=v^{\frac{3}{4}} / \varepsilon^{\frac{1}{4}} \quad \text { with } \quad \varepsilon=0.09 k \omega
$$

\section{References}

1. Batten, P., Goldberg, U., Chakravarthy, S.: Interfacing statistical turbulence closures with large eddy simulation. AIAA J. 42, 485-492 (2004)

2. Barone, M.F., Roy, C.J.: Evaluation of Detached Eddy Simulation for turbulent wake applications. AIAA J. 44, 3062-3071 (2006)

3. Durao, D.F.G., Heitor, M.V., Pereira, J.C.F.: Measurements of turbulent and periodic flows around a square cross section cylinder. Exp. Fluids 6, 298-304 (1988)

4. Fasel, H.F., Seidel, J., Wernz, S.: A methodology for simulations of complex turbulent flows. J. Fluids Eng. 124, 933-942 (2002)

5. Hsieh, K.J., Lien, F.S., Yee, E.: Towards a uniformed turbulence simulation approach for wall bounded flows. Flow Turbulence Combust 84, 193-218 (2010)

6. Israel, D.M.: A new approach for turbulent simulations in complex geometries. Ph.D. thesis, University of Arizona (2005)

7. Johansen, S.T., Wu, J.Y., Shyy W.: Filter-based unsteady RANS computations. Int. J. Heat Fluid Flow 25, 10-21 (2004)

8. Langhe, C. De, Merci, B., Dick, E.: Hybrid RANS/LES modelling with an approximate renormalization group. I: model development. J. Turbulence 6, 1-18 (2005)

9. Liu, N.S., Shih, T.H.: Turbulence modeling for very large eddy simulation. AIAA J. 44, 687$697(2006)$

10. Luo, S.C., Yazdani, M.G., Chew, Y.T., et al.: Effects of incidence and afterbody shape on flow past bluff cylinders. J. Wind Eng. Ind. Aerodyn. 53, 375-399 (1994)

11. Lyn, D.A., Einav, S., Rodi, W., et al.: A laser-Doppler velocimetry study of ensembleaveraged characteristics of the turbulent near wake of a square cylinder. J. Fluid Mech. 304, 285-319 (1995)

12. Magnient, J.C., Sagaut, P., Deville, M.: A study of built-in filter for some eddy viscosity models in large eddy simulation, Phys. Fluids 13, 1440-1449 (2001)

13. Moser, R.D., Kim, J., Mansour N.N.: Direct numerical simulation of turbulent channel flow up to $R e_{\tau}=590$. Phys. Fluids 11, 943-945 (1999)

14. Nicoud, F., Ducros, F.: Subgrid-scale stress modelling based on the square of the velocity gradient tensor. Flow Turbulence and Combustion 62, 183-200 (1999)

15. Peltier, L.J., Zajaczkowski, F.J.: Maintenance of the near-wall cycle of turbulence for hybrid RANS/LES of fully-developed channel flow. In 3rd AFOSR International Conference on Direct Numerical Simulation and Large-Eddy Simulation. Kluwer Academic (2001)

16. Sagaut, P., Deck, S., Terracol, M.: Multiscale and multiresolution approaches in turbulence. Imperial college press, London (2006)

17. Sohankar, A., Davidson, L.: Large Eddy Simulation of Flow Past a Square Cylinder: Comparison of Different Subgrid Scale Models. J. Fluids Eng. 122, 39-47 (2000)

18. Speziale, C.G.: Turbulence modeling for time-dependent RANS and VLES: a review. AIAA J. 36, 173-184 (1998)

19. Wilcox, D.C.: Turbulence modeling for CFD. 2nd Ed., DCW Industries, Inc. (2004)

20. Zhang, H.L., Bachman, C.R., Fasel, H.F.: Application of a new methodology for simulations of complex turbulent flows. AIAA-2000-2535 (2000) 